\title{
Focus on social neuroscience
}

\author{
We present a special focus on social neuroscience, bringing together several strands of research to highlight recent \\ progress in the field.
}

$\mathrm{t}$ is a truism that humans are a social species. But how does the brain implement social processes and behavior, and how do these processes in turn modify the brain? Despite the importance of social interactions to humans, this is an area that has traditionally been ignored by neuroscience, perhaps because interactions with complex social processes can sometimes be difficult to dissect and study in a lab setting. More recently, however, there has been an increasing interest in this area. Coupled with the willingness to borrow techniques from disciplines outside neuroscience, this has led to something of an explosion of research in social neuroscience. Here we focus on this topic with a collection of reviews, perspectives and a commentary that highlights some of the main topics in this field.

Social neuroscience is a relatively young field. Since the 1990s, we have seen an expansion of this field, and there are several dedicated journals and at least two societies aimed solely at advancing it. From its inception, social neuroscience has been a wide, interdisciplinary enterprise, and the articles in this special collection reflect the breadth of techniques used, ranging from animal work to human neuroimaging to genetics and behavioral analyses. On page 681 of this issue, Tania Singer and Cade McCall delve into both animal and human work to explore how the neuroendocrine system modulates social behavior, spanning social affiliation to aggression. They focus on three hormones that exemplify the far-reaching effects of the neuroendocrine system: oxytocin, arginine vasopressin and testosterone. In particular, this review critically discusses how the animal work can inform human research and suggests ways to advance future work.

Naomi Eisenberger and Steve Cole also appraise multidisciplinary work in their perspective on page 669, discussing the close ties between mental health and physical health. They describe the ways in which neurophysiological processes respond to social connection and disconnection to influence physical health outcomes: socially well connected people live longer on average and are more resistant to a variety of diseases. They suggest that threats to social connections may tap into the same alarm system that responds to threats that are critical to survival. Likewise, social connections may use a separate and mutually inhibitory system related to reward.

In a complementary perspective article, Andreas Meyer-Lindberg and Heike Tost discuss how social factors in turn modulate the risks for mental disorders. They bring together work from epidemiology, social psychology, neuroscience and genetics to describe the neural systems that are likely to implement this modulation. Psychiatric patients frequently have social deficits, and such deficits are good predictors of long-term disability. Conversely, rates of such disorders are increased in people with a history of adverse social environments, underlining the close relationship between social functioning and psychiatric disorders. However, such social factors have been largely ignored by most neuroscience work, in favor of clear genetic risk factors. Although such genetic risk factors are likely to be important, the authors emphasize the need to bring this strand of research together with the work on social connections, in order to identify potential converging mechanisms through which both kinds of risk factor may lead to psychiatric disorders.

The strength of these risk factors is not set in stone, however: the plasticity inherent in the nervous system means that interventions of various types result in functional and structural changes in the brain, with concomitant changes in behavior. The review by Richard Davidson and Bruce McEwen on page 689 describes how both negative and positive social factors, ranging from stress to meditation, affect brain structure and functioning. Both good and bad experiences seem to be effective in inducing plasticity, with animal work suggesting, for example, that both environmental enrichment and social stressors such as an unknown intruder lead to structural plasticity. However, the growing body of human work that suggests that things such as physical exercise, cognitive therapy and mediation can all result in central nervous system alterations that support positive behavioral outcomes raises the possibility that some of this plasticity may be used for therapeutic purposes, underlining the real-world applicability of this discussion.

A commentary by Joshua Buckholtz and René Marois, on page 655, also underlines how social neuroscience, perhaps more than any other part of neuroscience, has direct, real-world implications. The authors suggest that humans are unique in having stable and widespread cooperation among very large and genetically heterogeneous groups, which is very different from the scale on which prosocial behavior occurs in other animals, including other primates. What enables such 'ultra-sociality'? This commentary proposes that the ability to establish and enforce social norms is what underpins this otherwise puzzling phenomenon. Under this proposal, third-party punishment of social norm violations, the cornerstone of the criminal justice system, is especially crucial for stable cooperation, and the authors describe the potential neural and cognitive processes that are likely to underlie the learning and enforcement of social norms.

Finally, as with all newly established fields (and indeed, frequently, longestablished ones as well), there are still many questions and controversies, some of which have been the focus of much public attention. On page 675, Jamil Zaki and Kevin Ochsner offer a critical perspective on recent research on the neural systems supporting empathy, a key social function. They suggest that empathy can be understood as comprising two separate subprocesses, and encourage the use of more ecologically valid paradigms and an interpretation of brain activity grounded in behavioral outcomes.

There will no doubt be further controversies and pitfalls in this field as it matures, but we hope that the pieces in this special focus will give our readers a sense of recent research in social neuroscience, as well as inspire future work. As always, we welcome your feedback. 